\title{
Methods of substitution detected anomalous values in the realization of a random process
}

\author{
Vladimir I. Marchuk*, and Dmitriy Y. Chernyshov \\ Don State Technical University, 346500 Shakhty, Russia
}

\begin{abstract}
The paper considers a proposed method of detecting anomalous values in the realization of a random process in case of a limited amount of a priori information about the statistical characteristics of the noise component. A new approach is proposed to replace the detected anomalous values, which does not introduce a correlation relation into the results of the output pro-cessed random process.
\end{abstract}

\section{The statement of the problem}

Anomalous errors refer to large errors (type of miss), the value of which in some cases commensurate with the value of the measured physical process, and in some cases exceed it. They present only in some measurement results, with uncertain probability, but can take values comparable to the scale of the measurement message [1]. A fairly general and rigorous mathematical definition of normal and anomalous errors is currently absent, since the statistical characteristics of these errors depend on the conditions and causes of their occurrence, which are very diverse [1]. Data preprocessing (sampling from the General aggregation) includes the analysis of the sample size, noise of the sample, the analysis stands out sharply observed, independence of sample elements, and several others. The methods of nonparametric statistics (removal of noise, testing of statistical hypotheses and formation of requirements for the conditions of the experiment) are widely used.

In the presence of random non-stationary input signals, the use of classical statistical criteria for the detection of anomalous measurements is impossible. In this regard, the use of new methods can lead in some cases to a more effective solution for the problem of detection of anomalous measurements with a non-parametric priori uncertainty and a limited amount of measurement results. The use of the method of multiplication of estimates of the useful component [2] make it possible to develop a new adaptive method for detection anomalous measurements [3].

\section{Proposed method}

When choosing a threshold value in the classical approach, the probability of false alarm $\alpha$ and the probability of correct detection $\mathrm{D}$ are rigidly connected with each other, i.e., an increase in one leads to an increase in the other and Vice versa. The adaptation of the

*Corresponding author: marchuk@sssu.ru 
threshold is based on an automatic procedure for changing the threshold values depending on the estimation of the variance of the additive noise component [4]. The use of known methods is possible depending on the amount of a priori information about the analysed sample, which is shown in [4].

Reducing the priori information leads to a sharp decrease in the number of methods to solve the problem. Let us consider some results of the proposed method [3]. Figure 1 shows the dependency of the false alarm probability on the algorithm parameters.

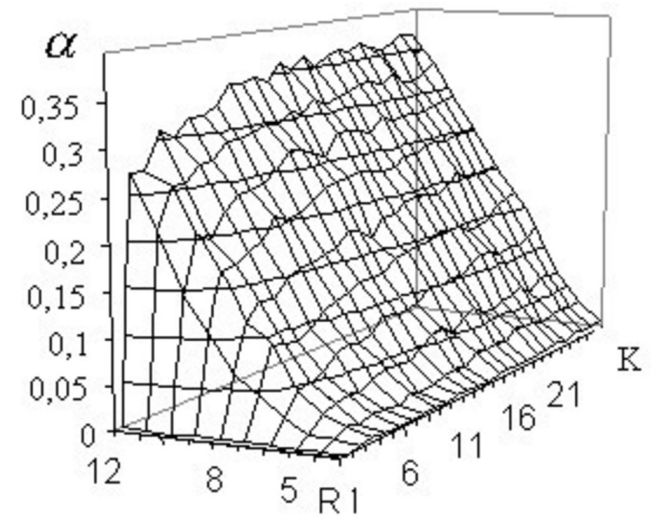

Fig. 1. Dependency $\alpha=f(R, K)$ for random sampling Gaussian stationary signal.

As follows from the analysis of the graph, the probability of an error of the first kind with an increase in the value of $R$ (number of coverages per sample length) increases, and at values when $K \geq 10$ (number of estimate multiplications) its value does not change, regardless of the value of $R$. It should be noted that the value of $\alpha=f(R, K)$ at the same time, it tends to zero. To generalize the results obtained, we consider the dependency of the probability of correct detection $\beta=f(R, K)$ from the same parameters of the algorithm, which is shown in Fig. 2.

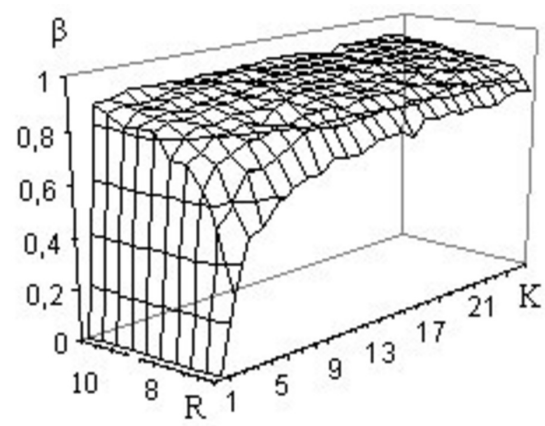

Fig. 2. Dependency $\beta=f(R, K)$ for random sampling Gaussian stationary signal.

The analysis of the research results, which are presented in Fig. 2 shows that the value of $K \geq 10$ value of $\beta$ practically does not change. At value of $R \geq 8$ the probability of correct detection reaches its maximum value $\beta \approx 0,9$. Therefore, on the basis of the conducted researches it is possible to recommend for practical use, in case of processing of realizations of casual stationary signals, to choose values $K \approx 10$ and $R \approx 8$, under which $\beta \approx 0,9$ and $\alpha \approx 0,01$. 
Fig. 3 shows the results of the study of the dependency according to $\alpha=f(\mathrm{D}, \mathrm{L})$, while fig. 4 according to $\beta=f(D, L)$ for the case when the initial realization represents a sample from the realization of a random stationary signal having a Gaussian distribution law with a variance value $\sigma_{\mathrm{mI}}=0,2$.

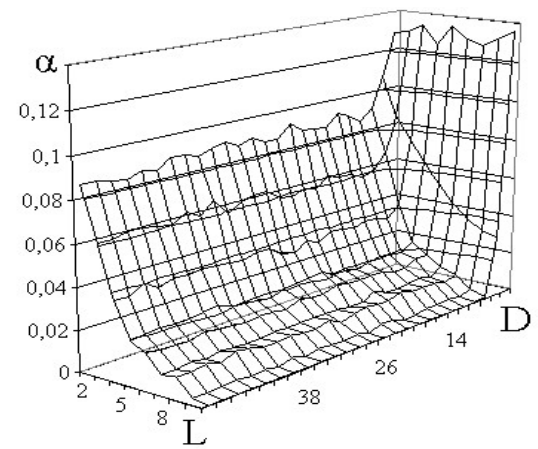

Fig. 3. Dependency $\alpha=f(D, L)$ with a uniform allocation of anomalous measurements along the sample.

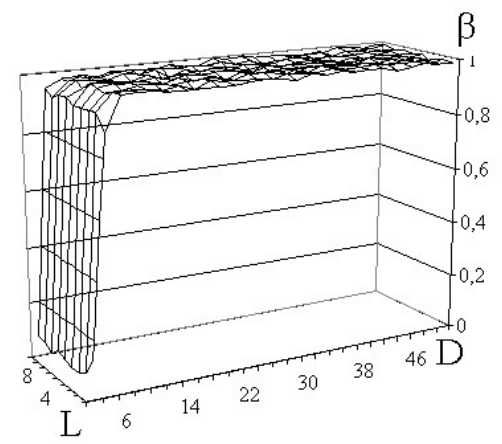

Fig. 4. Dependency $\beta=f(D, L)$ with a uniform allocation of anomalous measurements along the sample.

It is assumed that the value of the noise component dispersion (variance) remains constant along the entire sample. Anomalous measurement results are located evenly along the entire sample and replace the original measurement results.

\section{The obtained results}

The analysis of the obtained dependencies shows that with a uniform allocation of the anomalous measurement results, the error of the first kind decreases sharply with the increase in the number of anomalous measurements and practically does not depend on the amplitude at values $D \geq 6 \sigma_{\mathrm{m}}$. Probability of correct detection at values $D \geq 6 \sigma_{\mathrm{m}}$ also, it does not depend on the number of anomalous measurements and their amplitude and is almost equal to one, so the proposed method makes it possible to determine practically all emerging anomalous measurements. Thus, the effectiveness of the proposed method in detecting anomalous measurements located uniformly along the investigated realization is quite high. The results showed that the effectiveness of the method in detecting anomalous measurements does not depend on their location in the sample, as well as on values $D \geq 8 \sigma_{\mathrm{m}}$ and the value of their amplitude. A similar conclusion can be made when the dispersion of 
the noise component changes according to a linear and more complex law (for example, harmonic).

The proposed method for detecting anomalous measurements allows us to determine groups with a duration of no more than $2 \div 3$ measurements with a probability close to one, regardless of whether the dispersion of the noise component is constant or changes in a linear (or more complex) law. In both cases, regardless of the location of the group anomalous measurements (in the middle, at the end or at the beginning of the sample), the effectiveness of the proposed method does not change [4].

Determination of anomalous measurements in the sample of the process under study poses another problem, what to do with them. In practice, two methods are usually used, the first one is removing an anomalous value from the sample, which leads to a decrease in the sample (if the sample is small, this is unacceptable); the second one is replacing the average value from a number of standing values, which leads to a correlation of the sample values. In this regard, the following algorithm is proposed to replace the anomalous value. The anomalous values are determined, eliminated from the initial sample, the differential distribution function is estimated, a sequence of random numbers is formed according to the law of the obtained distribution function, by which the anomalous values are replaced by values whose probability in the evaluation of the differential distribution function differs from the estimate obtained.

The proposed technique allows to replace certain anomalous measurements without making a correlation between the samples and without reducing the sample length. Studies have shown that the proposed method is particularly effective if the number of anomalous measurements exceeds $10 \%$ of the sample length.

\section{Conclusion}

The proposed detection method makes it possible to detect anomalous measurements with a probability not worse than $\beta \approx 0,9$, the probability of false alarm does not exceed $\alpha \approx 0,01$.

The proposed method of elimination of anomalous measurements, as shown by the studies, has a high efficiency with the number of anomalous measurements of more than $10 \%$ of the length of the analyzed sample.

\section{References}

1. A.F. Fomin, O.N. Novoselov, A.V. Plyushchev, Rejection of anomalous measurements (Energoatomizdat, Moscow, 1985)

2. V.I. Marchuk, A.M. Muratov, Elements of receiving and amplifying devices: Interuniversity thematic collection (Taganrog, TRTI), 1, 87 (1982)

3. V.I. Marchuk, I.S. Shraifel Methods of isolating the signal component with a priori uncertainty and limited volume measurements: monograph (Publishing House of the SPSU, Saint-Petersburg, 2008)

4. V.I. Marchuk, K.E. Rumyantsev, Journal of the Russian Universities: Radioelectronics, 1, 20 (2006)

5. V.I. Marchuk, A.I. Sherstobitov, V.V. Voronin, S.V. Tokareva, Patent RU2301445, publ. 20.06.07, № 17.

6. V.I. Marchuk, A.I. Sherstobitov, V.V. Voronin, S.V. Tokareva, Patent RU2302655 publ. 10.07.07, № 19 . 